\title{
Potencial de biogás de los residuos agroindustriales generados en el departamento de Cundinamarca
}

\author{
Biogas potential of agro-industrial residues \\ generated in the department of Cundinamarca
}

\section{Potencial do biogás dos residuos agro-industriais gerados no departamento de Cundinamarca}

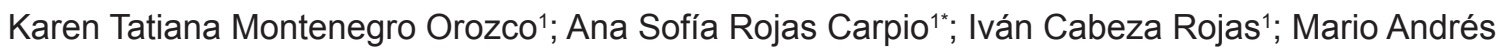 \\ Hernández Pardo ${ }^{*}$ \\ ${ }^{1}$ Facultad de Ingeniería Ambiental. Universidad Santo Tomás (USTA), Carrera 9 No. 51-11, Bogotá, Colombia \\ ${ }^{2}$ Facultad de Ingeniería. Universidad EAN, Calle 79 No. 11-45, Bogotá, Colombia \\ *mahernandez@universidadean.edu.co
}

Fecha Recepción: 22 de noviembre de 2015 Fecha Aceptación: 15 de julio de 2016

\begin{abstract}
Resumen
El bajo grado de tecnificación de la mayoría de las granjas en el departamento de Cundinamarca, conlleva a una alta tasa de generación de residuos agroindustriales, haciendo indispensable la evaluación de alternativas de valorización. En este trabajo se determinó el potencial de producción de biogás de los residuos agroindustriales en el departamento de Cundinamarca a través de digestión anaerobia. El potencial se estableció a partir de tres acercamientos metodológicos que incluían la evaluación de la generación de residuos en cada uno de los municipios, cálculo del potencial por tipo de biomasa residual en el departamento e identificación de tres regiones con el mayor potencial de producción de biogás. El cálculo del potencial se soportó en una recopilación, estandarización y procesamiento de la información disponible de áreas cultivadas y residuos generados en cada sector y/o actividad. Cundinamarca tiene un potencial promedio de biogás de 1.117.567TJ/año calculado por la transformación de los residuos agroindustriales a través de proceso anaerobio. Los residuos de mayor aporte en el potencial encontrado corresponden a aquellos generados por actividades relacionadas con los cultivos de café, caña panelera, arveja, papa; excretas bovinas y avícolas. Las regiones que podrían cobijar sistemas centralizados se identificaron en torno a los municipios de Fómeque, Guachetá y Anapoima, los cuales presentaron mayor potencial. El potencial estimado para estas regiones fue de 89.651, 127.513, 58.541TJ/año para la región 1 , región 2 y región 3 , los cuales son similares a los reportados para plantas de biogás en varias regiones del mundo.
\end{abstract}

Palabras clave: Biogás, biomasa, co-digestión anaerobia, potencial de biogás, residuos agro-industriales.

\begin{abstract}
The low level of technology in the farms of the Cundinamarca department, leads to a high rate of agroindustrial waste generation, making important the assessment of valorization alternatives. In this work, the biogas production potential of agro-industrial residues in the department of Cundinamarca was established through anaerobic digestion. The potential has been established from three methodological approaches, including the assessment of waste generation in each of the municipalities, calculation of potential by type of residual biomass in the department and identification of three regions with the greatest potential for biogas production. The estimation of the potential was supported in the collection,
\end{abstract}


standardization and processing of the information available and cultivated areas of wastes generated in each sector and/or activity. Cundinamarca has an average potential of biogas of 1,117,567TJ/year calculated by the transformation of agro-industrial wastes through the anaerobic process. The greatest contribution to this value corresponds to those residues generated from crops of coffee, sugarcane, peas, potatoes and cattle and poultry manure activities. The regions that could host centralized systems were identified in the surroundings of the municipalities of Fómeque, Guachetá and Anapoima, which presented the greatest biogas potential and substrates generation. The potential associated to those regions was 89,$651 ; 127,513 ; 58,541 \mathrm{TJ} /$ year for the region 1 , region 2 and region 3 , which are similar to those reported for biogas plants in various regions worldwide.

Keywords: Agro-industrial wastes, anaerobic co-digestion, biogas, biogas potential, biomass.

\section{Resumo}

O baixo nível de tecnologia da maior parte das fazendas, no departamento de Cundinamarca, conduz a uma elevada taxa de geração de resíduos agro-industriais, tornando a avaliação de avaliação alternativa essencial. Neste trabalho, o potencial de produção de biogás de resíduos agro-industrial no departamento de Cundinamarca é determinada através da digestão anaeróbica. O potencial é estabelecida a partir de três abordagens metodológicas, incluindo a avaliação da geração de resíduos em cada um dos municípios, o cálculo do potencial de por tipo de biomassa residual no departamento e identificação de três regiões com maior potencial para produção de biogás. O cálculo do potencial é suportado em uma coleção, padronização e processamento de superfícies de informação disponíveis e resíduos gerados em cada sector e/ou atividade. Cundinamarca tem uma média de biogás potenciais 1.117.567TJ/ano, calculado pela transformação de resíduos agro-industrial através de processo anaeróbio. Os maiores resíduos de contribuição encontrados no correspondem potencial para aqueles gerados pelas culturas afins de café, cana de açúcar, ervilhas, batatas atividades; gado e esterco de galinha. As regiões que poderiam cobrir sistemas centralizados foram identificados em torno dos municípios de Fómeque, Guachetá e Anapoima, que tiveram maior potencial. O potencial estimado para estas regiões foi de 89.651, 127.513, 58.541TJ/ano para a Região 1, região 2 e região 3, que são semelhantes aos relatados para as unidades de biogás em várias regiões do mundo.

Palabras-chave: Biogás, biomassa, biodigestão anaeróbia, resíduos agro-industriais, o potencial do biogás.

\section{Introducción}

En el departamento de Cundinamarca se generaron $727,7 \mathrm{t} / \mathrm{d}$ de residuos orgánicos representando un $66 \%$ de la generación total de residuos [1]. El hecho de que el departamento no cuente con una actividad agrícola completamente tecnificada, hace que la oferta de biomasa residual por hectárea sea alta $[1,2]$. Por otra parte, se tiene que la generación de residuos en el sector pecuario es importante, ya que la participación sectorial en el PIB es de 16,9\% [2], produciendo 23.024t/d de residuos [1]. Los residuos generados en estos sectores son dispuestos en su mayoría en los rellenos instalados para el municipio, los cuales se encuentran a distancias considerables desde el punto de generación, como es el caso del relleno nuevo Mondoñedo ubicado en Bojacá, praderas de Magdalena en Girardot y relleno sanitario doña Juana en Bogotá, lo que provoca un aumento en los costos de disposición y manejo de dichos residuos [3]. Teniendo en cuenta que el crecimiento económico que ha experimentado el país en los últimos años afecta directamente el sector agroindustrial aumentando el porcentaje de participación de los residuos de dicha actividad económica en la cantidad global generada [1]. Se hace necesario la evaluación técnica y económica de diversas tecnologías disponibles mundialmente con el fin de valorizar los mismos, aterrizando las mismas al entorno regional y nacional y realizando una transferencia ajustada a los sectores económicos que las requieren. La digestión anaerobia se ha establecido como una alternativa viable en el sector rural, teniendo en cuenta que tanto los residuos pecuarios como los agrícolas pueden ser empleados como materia prima para la generación de energía renovable que puede ser usada para cubrir las necesidades energéticas de la propia granja o ser vendida al mercado eléctrico, cuando el sistema de interconexión del país está adaptado a la generación distribuida [4]. Por otra 
parte, se ha demostrado que los procesos de codigestión maximizan el potencial de generación de biogás por lo tanto la mezcla de los residuos pecuarios y agrícolas puede ayudar a eliminar inconvenientes como la estacionalidad de los cultivos y la escasez de nitrógeno en los sustratos, el cuál es necesario para la cinética del proceso [4]. A través del proceso de co-digestión anaeróbica, la materia orgánica se descompone por acción de bacterias anaerobias desde su forma más compleja hasta su forma más simple produciendo biogás con un contenido de metano entre 55 y $70 \%$ [5]. Varios países son pioneros en la obtención de energía eléctrica a partir de la generación de biogás a través de procesos anaerobios como Alemania, Reino Unido y Dinamarca, esto indica que es una tecnología madura que puede ser transferida a los sectores industriales y rurales del país [6].

En el caso de Colombia el $65 \%$ de los residuos orgánicos generados de las actividades diarias son llevados dentro del paquete de residuos sólidos urbanos (RSU) a una disposición final en vertederos, celdas, rellenos u otro tipo de disposición [7], dejando de lado la implementación de técnicas para su aprovechamiento. El 35\% restante de residuos orgánicos son utilizados para su valorización en algunas zonas del país donde se estima que el $49 \%$ es utilizado para compostaje [8], $17 \%$ en procesos de aprovechamiento termoquímico, $13 \%$ en lombricultura [8,9], y un $21 \%$ en procesos de valoración agronómica [8]. Por su parte los procesos anaerobios en el país no son comúnmente utilizados, ya que sus aplicaciones han sido limitadas y a pequeña escala principalmente para el tratamiento de residuos provenientes de la actividad porcina y bovina donde se han utilizado biodigestores tubulares continuos en granjas industriales con un promedio de 2.300 cerdos y 1.300 reses [10]. Estos montajes han obtenido en promedio $500 \mathrm{~kW}$ los cuales son utilizados para satisfacer necesidades básicas de las granjas [10]. Esta práctica lleva en el país más de treinta años, utilizando la energía para necesidades básicas del sector rural como la cocción de alimentos e iluminación [11], lo que permite identificar el alto potencial que presentan este tipo de residuos para la generación de energía en instalaciones centralizadas que integren diversos productores.

Adicionalmente, la mayoría de los trabajos realizados por la comunidad científica internacional se han centrado en materias primas de países desarrollados, por lo que la biomasa proveniente de países en vía de desarrollo ha sido muy poco estudiada y en Colombia específicamente, no existe un censo completo a nivel regional que presente en detalle la disponibilidad y potencial de los residuos provenientes del sector agroindustrial $[1,4]$. De acuerdo a esto se hacen indispensables procesos de evaluación, cuantificación y caracterización de la biomasa residual que se genera en los procesos productivos, permitiendo el establecimiento de proyecciones adecuadas para la estimación del potencial de biogás a partir de residuos agroindustriales como fuente de energía. Por ende, el objetivo de este estudio es establecer el potencial de producción de biogás de los residuos provenientes de actividades agrícolas y pecuarias generados en el departamento de Cundinamarca, reconociendo los municipios y residuos que tengan mayor potencial de generación de biogás como resultado de sus tasas de generación o sus potenciales biológicos de producción de metano presentando tres posibles regiones las cuales son una opción viable para la valorización de este tipo de residuos por medio de procesos de co-digestión anaerobia. Esto soportado en una recopilación de información de producción de residuos, en los potenciales biológicos de metano de cada residuo y la disponibilidad espacial y temporal de los residuos. Esto se hace como un primer paso para el aprovechamiento de la biomasa residual en el marco de la Ley 1715 del 2014 sobre la integración de las energías renovables no convencionales al sistema energético nacional.

\section{Metodología}

El potencial de biogás a partir de biomasa residual fue establecido a partir de tres acercamientos metodológicos: el primeroseenfocó en la evaluación de la generación de residuos en cada uno de los municipios, el segundo estuvo orientado a calcular el potencial de biogás por tipo de biomasa residual en el departamento y por último se determinaron tres localizaciones donde se identificaron regiones que mostraron el mayor potencial de biogás en el departamento. Todos estos cálculos se soportaron en la recopilación de información relacionada con los sistemas de producción agrícola y pecuaria del departamento, tomando como fuente la información suministrada por la Gobernación de Cundinamarca en la subdirección de planeación y desarrollo rural, la información de la Encuesta Nacional Agropecuaria (2013), el anuario estadístico del sector agropecuario (2012), el 
censo nacional agropecuario (2014) suministrado por el Departamento Administrativo Nacional de Estadística (DANE), levantamiento de información en campo en la UMATA de los municipios. Los datos se validaron a través de métodos estadísticos como media, moda, variabilidad de parámetro y nivel de confianza con el fin de tener un grado de certidumbre mayor en los datos $[2,3,12,13]$.

La generación de residuos se determinó a partir de la información de los cultivos existentes y de las actividades pecuarias adelantadas en el departamento, donde las fuentes de información consultadas fueron el Atlas de la biomasa, consultas en la UMATA y revisión de bibliografía sobre generación y disposición de residuos. De acuerdo a cada cultivo, transitorio o permanente, se identificó la generación de residuos respecto a la producción del cultivo y la fracción de biomasa del proceso que se pierde como residuo. Esta fracción se discriminó por cada tipo de biomasa: hojas, ramas, tallos, etc. Por su parte, la generación de estiércol se determinó a partir del número de animales presentes en cada subsector pecuario. Al respecto se utilizaron tasas de generación de estiércol para bovinos, porcinos y aves discriminando las mismas por edad, tipo o propósito de los animales (Tabla 1).

Tabla 1. Tasa de generación de estiércol de acuerdo a cada subsector y el tipo de animal $[14,15]$.

\begin{tabular}{ccc}
\hline Subsector & Tipo & $\begin{array}{c}\text { Estiércol } \\
\text { (kg/animal/d) }\end{array}$ \\
\hline Bovino & Machos y hembras de 12 a 24 meses & 8 \\
& Machos y hembras de 24 a 36 meses & 10 \\
& Machos y hembras > de 36 meses & 15 \\
Porcino & Machos y hembras < de 12 meses & 20 \\
& Cría & 2 \\
Avícola & Levante & 2,35 \\
& Ciclo completo & 1,35 \\
\hline
\end{tabular}

El potencial de biogás (Ecuación 1) se determinó utilizando la estimación de la generación de residuos (agrícolas y pecuarios) de cada municipio teniendo en cuenta el rendimiento y estacionalidad y actividad pecuaria (Tabla 1). Adicionalmente, se identificaron las características físico-químicas de cada sustrato como porcentaje de sólidos volátiles $(\% \mathrm{SV})$, relación carbono/nitrógeno $(\mathrm{C} / \mathrm{N})$ y por último el potencial biológico de metano (BMP, por sus siglas en inglés). Por ende, el potencial de metano se obtuvo como el resultado del producto entre el BMP expresado en $\left(\mathrm{m}^{3} \mathrm{CH}_{4} / \mathrm{kgSV}\right)$, el contenido de sólidos volátiles de cada residuo ( $\mathrm{kgSV} / \mathrm{kg}$ residuo) y la generación de cada residuo (kgresiduo). Por su parte, el potencial energético (TJ/año) se determinó a partir del potencial de biogás y el poder calorífico del metano obteniendo la energía bruta contenida en el metano sin ningún ajuste al rendimiento de los aprovechamientos energéticos. Con esta información se obtuvo el potencial de biogás y el potencial energético por residuo, municipio y regiones planteadas.

$$
\text { Potencial de metan } o=\text { Generación de residuos } * \% \text { Solidos Volátiles } * \text { BMP }
$$

La determinación de las tres localizaciones para la instalación de plantas de biogás en algunas regiones de Cundinamarca se soportaron en la distribución de los sustratos que cuentan con un mayor potencial de biogás, la generación de residuos, distancia entre puntos de generación de los residuos y los costos de transporte relacionados con dicha distancia. Se empleó el software Arcgis para obtener la distancia entre cada uno de los municipios, para la distribución de dichas zonas se tomó una distancia la cual oscila entre 10 y $30 \mathrm{~km}$ entre cada punto seleccionado, debido a que a una distancia menor a $10 \mathrm{~km}$ y mayor a $30 \mathrm{~km}$ aumentaría los costos variables asociados al transporte. El cálculo del costo de referencia para el transporte de carretera se estimó usando el software del Ministerio de Transporte: sistema de información de costos eficientes para el transporte 
automotor de carga. En donde para una distancia menor a $10 \mathrm{~km}$ los costos asociados al transporte serían de $\$ 3.313$ por kilómetro y para una distancia mayor será de \$2.972 por kilómetro [13].

\section{Análisis de Resultados}

\section{Actividad pecuaria y agrícola en el departamento de Cundinamarca}

De acuerdo con los datos recolectados en la encuesta agropecuaria (2013), se consolidó que hay 246.106ha cosechadas, con un total de 121.188ha de cultivos permanentes y 118.979ha de cultivos transitorios. Los cultivos transitorios ocupan un área de 130.834ha sembradas con una producción estimada de $1.589 .498 \mathrm{t}$, resaltando los cultivos de papa, arveja y maíz como mayores productores. Los cultivos permanentes presentan áreas sembradas de 137.215ha, con una producción estimada de 735.299t donde los cultivos de caña, café, flores y mora son los mayores productores en el departamento. Respecto a los residuos agrícolas se tienen en cuenta los asociados a cosecha que representan hojas, ramas, cáscara, fibra, pulpa, entre otros; para la estimación de la cantidad de residuos generados por cada cultivo se tuvo en cuenta el porcentaje de biomasa residual de cada residuo y la producción de cada cultivo. Los cultivos permanentes y transitorios que predominan en el departamento son flores, caña, cacao, papa, entre otros (Tabla 2).

Tabla 2. Generación de biomasa residual de los principales cultivos de Cundinamarca.

\begin{tabular}{|c|c|c|c|c|}
\hline $\begin{array}{l}\text { Tipo de } \\
\text { cultivo }\end{array}$ & Cultivo & Tipo de biomasa & $\begin{array}{c}\% \text { de } \\
\text { biomasa }\end{array}$ & $\begin{array}{l}\text { Generación de } \\
\text { biomasa(t/año) }\end{array}$ \\
\hline \multirow{11}{*}{ Permanente } & \multirow{2}{*}{ Flores } & Hojas de poda & 39 & 77.175 \\
\hline & & Ramas secas & 26,7 & 3.743 \\
\hline & \multirow{2}{*}{$\begin{array}{c}\text { Caña } \\
\text { panelera }\end{array}$} & Caña & 35 & 64.130 \\
\hline & & Bagazo & 63 & 115.434 \\
\hline & Palma & Fibra de palma & 14 & 11.110 \\
\hline & \multirow[t]{2}{*}{ Banano } & Pinzote & 35 & 14.850 \\
\hline & & Cisco & 20 & 8.486 \\
\hline & \multirow[t]{2}{*}{ Café } & Pulpa & 15 & 6.364 \\
\hline & & Mucílago & 4 & 1.143 \\
\hline & \multirow{3}{*}{ Cacao } & Cáscara & 65 & 17.008 \\
\hline & & Hojas de poda & 5 & 1.031 \\
\hline \multirow{10}{*}{ Transitorio } & & Hojas de poda & 39 & 15.148 \\
\hline & \multirow[t]{2}{*}{ Mora } & Ramas mora & 10 & 31.395 \\
\hline & & ramas secas & 26,7 & 77.134 \\
\hline & \multirow[t]{2}{*}{ Arveja } & Hojas de poda & 39 & 8.758 \\
\hline & & Chalas & 8,9 & 383 \\
\hline & \multirow{3}{*}{ Maíz } & Ensilaje & 12 & 2.948 \\
\hline & & Caña & 20 & 30.066 \\
\hline & & Rastrojos & 17,6 & 29.995 \\
\hline & Papa & Residuos de papa & 25 & 32.487 \\
\hline & Frijol & Hojas y tallos & 48 & 25.223 \\
\hline
\end{tabular}

Los residuos generados a partir de estos cultivos dependen del rendimiento de cada uno, donde según las estadísticas agropecuarias realizadas por la Gobernación de Cundinamarca, los cultivos transitorios generan en promedio $55 \%$ del total del cultivo como residuo; referente a los cultivos permanentes se estima que solo se aprovecha un $35 \%$ del total del cultivo. Esta información es vital para la optimización de los sistemas buscando minimizar costos, transporte, almacenamiento y aspectos ambientales [16]. Dentro de la información analizada, los cultivos de rosas, caña 
panelera, palma, banano y café son los de mayor producción en el departamento, pero tan solo los cultivos de rosas y café presentan una mayor tasa de generación de residuos siendo ésta alrededor de $50 \%$ y $69 \%$, respectivamente. Por otro lado, en el departamento se resaltan las actividades pecuarias donde el subsector bovino clasifica a las especies según su edad y propósito (Tabla 1). El subsector porcino se clasifica según su tipo de crianza (tradicional y tecnificada) y por último el subsector avícola se encuentra clasificada según su propósito (engorde, postura o traspatio). La cantidad de animales que estaban presentes en el departamento se obtuvieron a partir de las estadísticas agropecuarias presentadas por la Gobernación de Cundinamarca para el año 2013 (Tabla 3). En donde se evidencia que el departamento tiene una vocación fuerte hacia la industria avícola según el número de animales. Aunque en términos de generación de estiércol el principal aporte está dado por la actividad bovina.

Tabla 3. Generación de estiércol en Cundinamarca.

\begin{tabular}{ccc}
\hline Subsector & $\begin{array}{c}\text { Número de } \\
\text { animales }\end{array}$ & $\begin{array}{c}\text { Producción de } \\
\text { estiércol }(\mathbf{t} / \mathbf{d})\end{array}$ \\
\hline Bovino & 1.241 .834 & 18.627 \\
Porcinos & 735.860 & 1.729 \\
Avícola & 15.695 .129 & 2.668 \\
\hline
\end{tabular}

\section{Potencial de biogás por municipio}

Los resultados del potencial de biogás por municipio muestran que la biomasa residual pecuaria aporta en promedio el $76 \%$ del total frente al resto de residuos orgánicos, principalmente en los municipios encontrados en la Figura 1. Esto es coherente dada la generación de estiércoles que es de 981.120t/año, 631.085t/año y 6.798.855t/ año para aves, porcinos y bovinos, respectivamente [1] y por los potenciales de biológicos de metano de estos residuos $0,7 \mathrm{~m}^{3} / \mathrm{kgSV}, 0,351 \mathrm{~m}^{3} / \mathrm{kgSV}$ y $0,6 \mathrm{~m}^{3} / \mathrm{kgSV}$, respectivamente [17-19]. Sin embargo, estos valores deben revisarse identificando el estado de tecnificación de los procesos que conlleven a la recolección y disponibilidad de los residuos. Esta tendencia es diferente en el municipio de Suesca, ya que en este municipio el desarrollo de actividades agrícolas es el que aporta una mayor cantidad de residuos, por ello en su mayoría el potencial de biogás está dado por el desarrollo de cultivos permanentes principalmente de flores. Los municipios de Anapoima y Suesca presentan el mayor potencial de biogás producto de cultivos permanentes debido a que ocupan el $94 \%$ de hectáreas disponibles para la agricultura en el departamento. La presencia de cultivos como: mango, mandarina, naranja, lima, caña panelera, cacao, café y flores incrementa la generación de residuos dadas las tasas por área que son de 2.218.516t/ha [1] en el municipio de Suesca. Dado que los sólidos volátiles y el potencial biológico de metano tienen una relación directamente proporcional, se tiene que los cultivos mencionados anteriormente tienen un promedio del $94,6 \%$ de sólidos volátiles lo cual favorece la producción de metano [20]. Por el contrario, para el cultivo de caña panelera, el bagazo resultante tiene un contenido bajo de sólidos volátiles; entre 30 y $38,5 \%$ [21,22]. Para el cacao, los residuos (poda y cáscara) tienen un bajo potencial biológico de metano, pero son importantes debido al nivel de generación en el municipio. Adicional a ello se establece que el alto potencial de biogás que presenta el municipio de Suesca se encuentra relacionado con la alta producción de claveles y rosas la cual es de 32.900.040t en 90ha, esto aumenta la cantidad de residuos generados. Por último, la generación de residuos durante el beneficio del café corresponde al $95 \%$ del cultivo representado en pergamino, pulpa, mucílago y cisco [23], entre estos materiales el mucílago y la pulpa pueden aprovecharse para la producción de metano [24].

En los cultivos transitorios y permanentes se tiene que durante la poda y/o mantenimiento se generan alrededor de 15 a $25 \%$ de los residuos, los cuales corresponden a hojas, tallos y producto en descomposición o defectuoso [25,26]. Aunque estos materiales son principalmente lignocelulósicos, los sólidos volátiles pueden ser elevados como para el caso del cultivo de arveja donde se alcanza un $60 \%$, cebada $77 \%$, papa $78 \%$ y tomate con $93 \%[27,28]$. Tibirita muestra un alto potencial de biogás asociado a la existencia de cultivos transitorios los cuales ocupan un 51\% de hectáreas disponibles para siembra, allí se encuentran cultivos como arveja, frijol, habichuela, papa, tomate y ahuyama generando $4.851 \mathrm{t}$ de residuos. Sin embargo, la dispersión de la producción a lo largo del municipio es una limitante para el aprovechamiento a gran escala de estos residuos. Sin embargo, es punto de partida para la implementación de sistemas a pequeña escala los cuales tienen una operación y mantenimiento 
sencillo y sus desempeños ambientales y económicos son mejores comparados con sistemas a mediana y gran escala [29].

El mayor potencial de biogás generado por actividades pecuarias (Figura 1) se encuentra en el municipio de Fómeque, donde registran 5.115 bovinos, entre los 12 y 36 meses que producen un total de $51 \mathrm{t} / \mathrm{d}$ de estiércol, 11.826 cabezas porcinas generando $27 \mathrm{t} / \mathrm{d}$ de estiércol y 962.416 de aves generando 158t/d de estiércol. En el municipio de Gachalá se tienen
8.300 bovinos entre machos y hembras de 12 a 36 meses generando $83 \mathrm{t} / \mathrm{d}$ de estiércol, en el sector porcino existen 2.346 cabezas en granjas tradicionales generando $5 \mathrm{t} / \mathrm{d}$ de estiércol, en el sector avícola hay 1.303 .400 aves generando $221 \mathrm{t} / \mathrm{d}$ de estiércol [12].

De acuerdo a esto, la cantidad de estiércol generadas dependerá de la edad del animal, ya que se estima que los animales entre los 24 y 36 meses podrán producir una mayor cantidad de estiércol dependiendo de la especie [30].

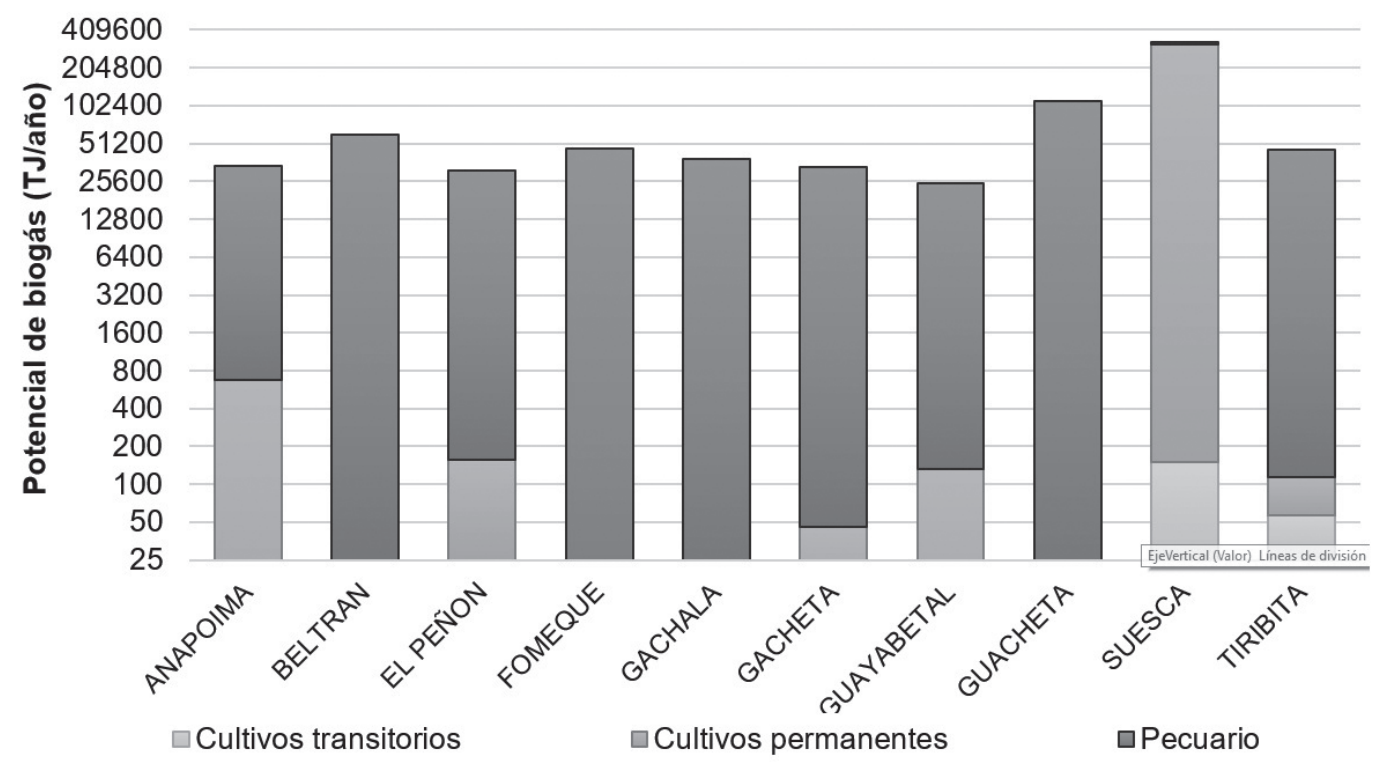

Figura 1. Distribución del potencial energético discriminado por el aporte de cultivos transitorios y permanentes y sector pecuario para los 10 municipios con mayor potencial de biogás.

\section{Identificación del potencial de biogás por residuo}

De acuerdo a la alta generación de residuos producidos por actividades agrícolas y pecuarias en el departamento, se estimó el potencial de biogás para cada residuo a manera de contrastar su importancia frente a la generación de residuos aprovechables. De acuerdo a esto (Figura 2) se logró establecer que, en función de la generación y el BMP, los residuos que mayor potencial presentan son los pecuarios y los generados por cultivos transitorios como el café, caña panelera, mora, rosa y clavel, arveja, frijol y papa. A partir de variables como generación de residuos de cada cultivo y la evaluación de BMP de cada sustrato se seleccionaron una serie de residuos con alto potencial de metano como pulpa, y mucílago obtenidos del cultivo de café, bagazo de caña resultado de la producción de caña panelera, hojas de poda recolectadas en el cultivo de rosas y claveles, hojas y tallos procedentes de los cultivos de mora, frijol, arveja y papa. El estiércol producido por especies avícolas, bovinas y porcinas presenta un alto potencial de biogás debido a diversos factores, entre ellos se encuentra el gran volumen de estiércol producido en el departamento. En donde la actividad avícola tiene la mayor presencia en el departamento destacando el mayor potencial de biogás para ser aprovechable.

La relación carbono/nitrógeno $(\mathrm{C} / \mathrm{N})$ es un factor determinante en la producción de metano ya que a valores bajos de $\mathrm{C} / \mathrm{N}$ incrementa el riesgo por exceso de nitrógeno y a su vez la inhibición del proceso, mientras que valores altos indican deficiencia de nitrógeno para la síntesis de biomasa [20]. Valores de $\mathrm{C} / \mathrm{N}$ entre 20-30 presentan valores positivos e incrementan el rendimiento y el potencial de metano (Tabla 4). La relación 
para el estiércol producido por especies bovinas, porcinas y avícolas es de 20 [22], por lo que estos residuos son capaces por si solos de mantener el proceso de digestión anaerobia. A su vez, las elevadas relaciones de SVIST (Tabla 4) garantizan disponibilidad de materia biodegradable para la producción de metano [18,31]. Sin embargo, el rendimiento de la producción de metano se puede ver afectado por la presencia de un alto porcentaje de proteínas ya que podrían ser responsables de la baja biodegradabilidad del residuo debido a los productos intermedios de su degradación [27]. En cuanto a los residuos vegetales de algunos cultivos, los elevados valores de $\mathrm{C} / \mathrm{N}$ y la escasez de elementos traza pueden afectar el correcto desarrollo del proceso por la producción de ácidos grasos y el decrecimiento del pH del sistema [32]. De acuerdo a esto, y en general, para contrarrestar las deficiencias de los diferentes residuos se deben plantear procesos de co-digestión que ayudan a incrementar la producción de metano respecto a los potenciales individuales [33]. En la co-digestión, los sustratos pueden estimular la síntesis de enzimas por la composición del material o la presencia de microorganismos que favorecen la sinergia del proceso [34].

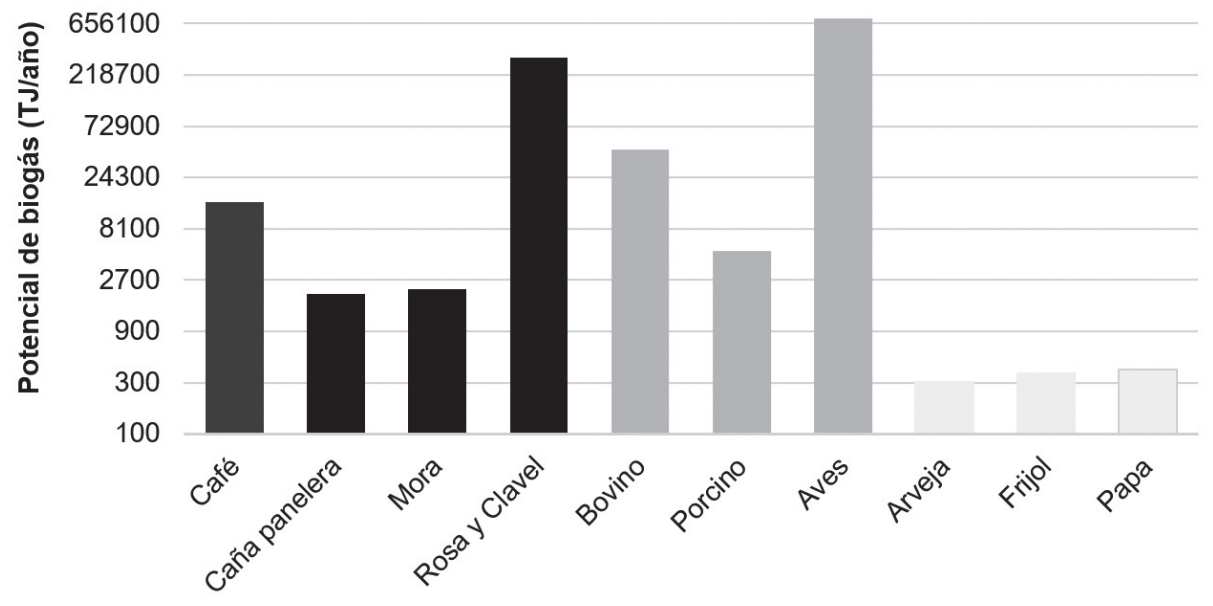

Figura 2. Potencial teórico de biogás por tipo de residuo en Cundinamarca.

Tabla 4. Características de los principales residuos generados.

\begin{tabular}{ccccc}
\hline \multirow{2}{*}{ Origen } & \multirow{2}{*}{ Residuos } & \multicolumn{3}{c}{ Características } \\
\cline { 3 - 5 } & & Relación C/N & SV/ST & \%proteínas \\
\hline Porcino [18] & Estiércol & 20 & 0,72 & 13,2 \\
Vacuno [18] & Estiércol & 20 & 0,75 & 17 \\
Aves [18] & Estiércol & 20 & 0,77 & 20,5 \\
Papa [35,36] & Hojas y tallos & 40 & 0,58 & 1,9 \\
Frijol [37] & Hojas y tallos & 12 & 0,93 & 15,2 \\
Arveja [37] & Hojas y tallos & 11 & 0,93 & 20 \\
Rosas y clavel [35,38] & Hoja de poda & 13 & 0,87 & 2,2 \\
& Mucílago & 20 & 0,68 & 21,4 \\
Café [39,40] & Pergamino & 20 & 0,40 & 6,4 \\
& Bagazo de caña & 150 & 0,43 & 20 \\
Caña panelera [41] & Caña & 21 & 0,80 & 15,3 \\
\hline
\end{tabular}




\section{Distribución regional}

La selección de las regiones (Figura 3) se realizó involucrando como variable principal municipios con elevado potencial de biogás. De acuerdo a esto, el elevado potencial de biogás calculado para municipios como Fómeque para la región uno, Guachetá para la región dos y Anapoima para la región tres, los hizo candidatos para el planteamiento de las regiones alrededor de ellos. La región uno está conformado por los municipios de Choachí, Fómeque, Gachalá y Junín. En la región dos se encuentran los municipios de Guachetá, Fúquene, Lenguazaque, Ubaté y Susa. Por último, la región tres está constituida por Anapoima, El Colegio, La Mesa y Viotá. Otra de las variables involucradas es la distancia entre municipios oscilando en un rango de 10 a $30 \mathrm{~km}$ entre el municipio de mayor potencial como punto central y los municipios aledaños a éste. En la región número uno se estima una distancia de $25 \mathrm{~km}$ entre el municipio de Fómeque y Gachalá, $30 \mathrm{~km}$ entre Fómeque y Junín, $20 \mathrm{~km}$ y $29 \mathrm{~km}$ desde Choachí hasta el punto central de dicha región. En la región número dos se encuentra a una distancia de $11,4 \mathrm{~km}$ desde Fúquene a Guachetá, $19 \mathrm{~km}$ entre Guachetá y el municipio de Susa, desde el municipio de Ubaté y Guaduas hay una distancia de $18 \mathrm{~km}$, y $9 \mathrm{~km}$ desde Lenguazaque a Guachetá. Finalmente, para la región número tres se estima una distancia de $11 \mathrm{~km}$ desde el punto central a la mesa, $10 \mathrm{~km}$ entre El Colegio y Anapoima y $14 \mathrm{~km}$ desde Anapoima hasta Viotá. El potencial de cada una de las zonas regionales (Figura 4) se encuentra entre 31.800TJ/año y 127.949TJ/año, valores que están en los rangos de instalaciones implementadas en Alemania, España, Dinamarca y Polonia las cuales utilizan purines de especies bovinas y porcinas utilizando como co-sustratos residuos lignocelulósicos provenientes de cultivos de maíz, hojarasca, residuos de industrias de alimentos y residuos de lodo de depuradora; dichas instalaciones tienen en promedio un potencial energético de 104.179TJ/año [42-44].

El mayor potencial de biogás se obtuvo para la región 2 con 127.749TJ/año lo cual se vio influenciado principalmente por la generación asociada a las excretas avícolas (Figura 4). La generación de residuos en la región 1 varía de acuerdo a la disponibilidad y a las épocas de cultivos transitorios como zanahoria, maíz, frijol, habichuela, tomate y arveja. Adicionalmente, cultivos permanentes como caña panelera, aguacate, cacao, café y plátano tienen un gran aporte de residuos ya que se encuentran disponibles los 12 meses del año. Las actividades pecuarias también realizan un gran aporte en la generación de sustratos, debido a que la proporción de excretas de animales provenientes de granjas tecnificadas es alta, esta región cuenta con 8.848 bovinos y 3.104 porcinos en granjas tradicionales, 41.060 aves de traspatio, 4 granjas productoras de leche y 35 granjas productoras de carne bovina las cuales en su mayoría son tecnificadas, lo que permitirá una recolección eficiente de parte del residuo. Respecto a la región dos se encuentran cultivos permanentes como fresa, mora, uchuva y flores, cultivos transitorios como arveja, cebolla, maíz, papa y frijol, esta región cuenta con 293 granjas productoras de leche y 282 granjas productoras de carne. Finalmente, la región 3 presenta cultivos permanentes como mango, mandarina, limón café aguacate, banano, cacao y caña panelera, cultivos transitorios como maíz, arveja, tomate y frijol; encontrándose 825 granjas productoras de leche, 15 granjas productoras de carne y 24 granjas de engorde, 4 granjas de postura para un total de 15.000 aves de traspatio. En resumen, en la región 1 el municipio de Fómeque es el mayor generador de estiércol avícola lo cual se encuentra relacionado con el alto potencial de biogás de esta región. En la región 2 los municipios que mayor aportan sustratos provenientes de los sub sectores porcinos, avícolas y bovino son Fúquene, Guachetá y Ubaté respectivamente. En la región 3 sobresalen los municipios de Viotá con la mayor producción de excretas por actividades porcícolas y Anapoima por actividades avícolas. La distribución de residuos en cada central propuesta (Tabla 5) es coherente con la participación que se da en las centrales de biogás instaladas en varias regiones del mundo principalmente en España, donde el mayor potencial de biogás registrado en estas instalaciones fue de 421.142TJ/año el cual se obtuvo a partir del uso de estiércol de porcinos y aves en Andalucía y Melilla [42]. 

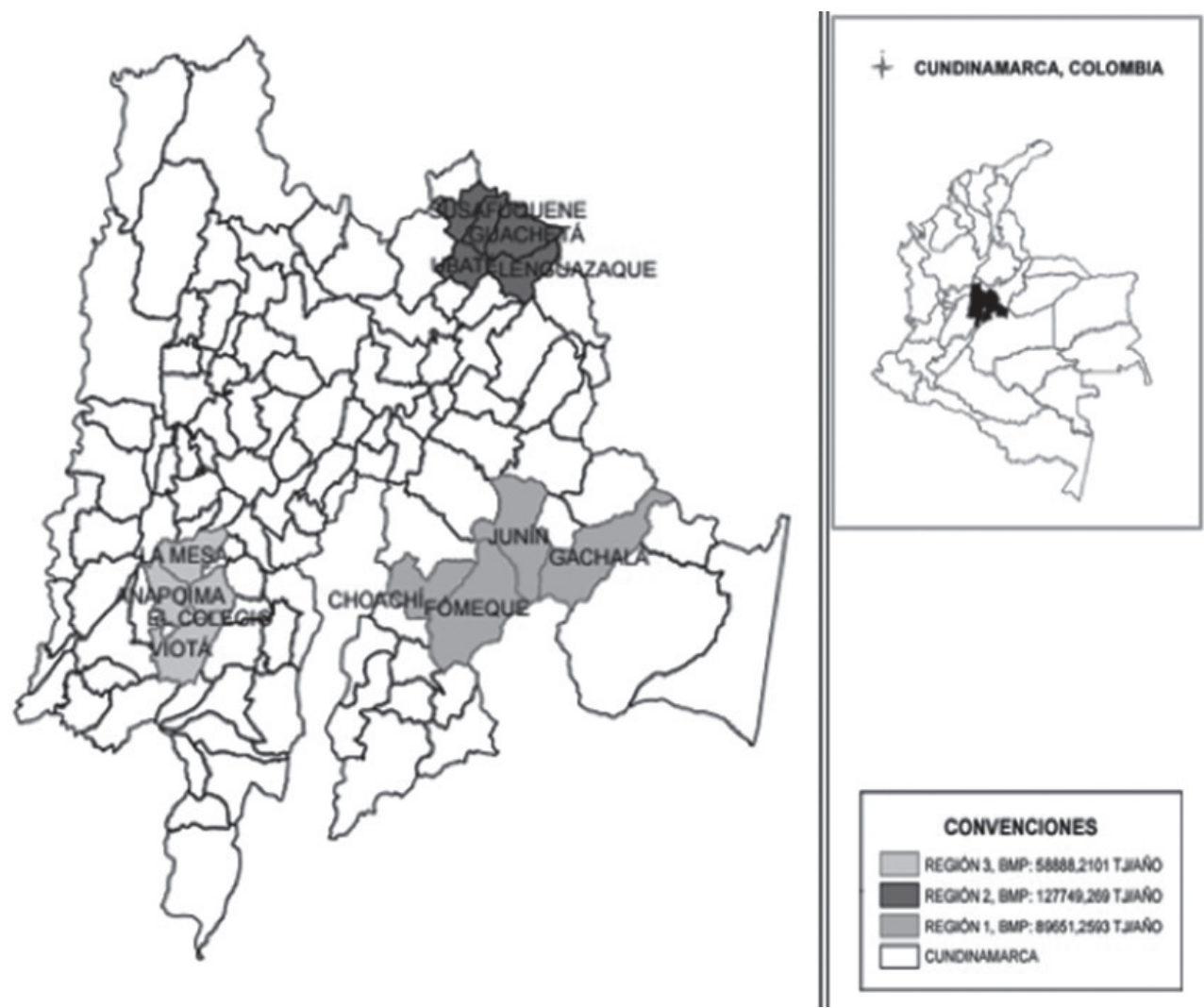

Figura 3. Mapa de Cundinamarca con la selección de puntos para el cálculo de potencial de metano por regiones.

Tabla 5. Potencial de biogás por tipo de residuo en cada región.

\begin{tabular}{cccc}
\hline \multirow{2}{*}{$\begin{array}{c}\text { Especies y } \\
\text { Cultivos }\end{array}$} & \multicolumn{3}{c}{ Potencial de biogás (TJ/año) } \\
\cline { 2 - 4 } & Región 1 & Región 2 & Región 3 \\
\hline Bovinos & $1.273,2$ & $1.819,6$ & 674,2 \\
Avícolas & $86.864,4$ & $125.322,4$ & $53.989,2$ \\
Porcinas & 412,7 & 158,2 & 591,8 \\
Permanentes & 139,9 & 9,9 & $3.285,7$ \\
Transitorios & 30,4 & 203,1 & 3,2 \\
Total & $88.720,6$ & $127.513,2$ & $58.544,1$ \\
\hline
\end{tabular}

Adicionalmente, para el aprovechamiento de los residuos existentes en las tres zonas, se podrá utilizar el proceso de co-digestión anaerobia con la utilización de reactores de cúpula fija y móvil. Estos sistemas por la sencillez de sus materiales para su construcción, son recomendables para granjas que pretendan producir biogás para autoconsumo [45]. Si por el contrario se pretende aprovechar una gran cantidad de residuos lignocelulósicos y estiércol será recomendable el uso de un digestor continuo con el fin de tener una operación constante. También se espera que la carga añadida a los reactores se mezcle en su totalidad debido a la heterogeneidad de los residuos, por ello será necesario la utilización de un biodigestor de mezcla completa o biodigestores tubulares [46]. Los costos para la elaboración de estos biodigestores no son altos, lo que permitirá que dicha tecnología esté al alcance de toda la población de las regiones nombradas [31]. Adicionalmente, durante la implementación de este tipo de tecnología es importante evaluar los impactos relacionados a la construcción, la operación, el transporte, el almacenamiento y el uso del digestato, entre otros. Ya que estás actividades generan emisiones de gases efecto invernadero que deben analizarse frente a las emisiones que naturalmente generan los residuos sin ningún tipo de manejo $[47,48]$.

La disponibilidad de los sustratos a lo largo del año es un factor fundamental para la viabilidad de la instalación de una planta de aprovechamiento. De acuerdo a esto, en la Figura 5 se muestra la dinámica del potencial de biogás asociada a la disponibilidad de los residuos acorde al tiempo, esto afecta a los cultivos sean transitorios 0 
permanentes. Mientras que la generación de estiércol se asume como constante a lo largo del año razón por la cual no afecta el potencial de biogás en el tiempo. Por esta razón y sumado al potencial de los residuos se encuentra que el potencial total de la región se ve ligeramente afectado por esta disponibilidad. Por lo tanto, los municipios de Guachetá, Fúquene, Lenguazaque, Susa y Ubaté, los cuales se encuentran clasificados en la región 2 , presentan potencial de biogás similar debido a la generación permanente de estiércol proveniente de especies bovinas, porcinas y avícolas. Mientras que se tiene un mayor aporte al potencial por parte de los cultivos transitorios a comparación de los permanentes principalmente en los meses de Junio, Julio y Octubre. La recolección de estiércol en esta zona es viable debido a que, según el censo agropecuario, el $70 \%$ de los animales presentes en esta región se encuentran en granjas tecnificadas. En caso que el sistema de digestión anaerobia dependa principalmente de los residuos de cultivos (transitorio/permanente) este se ve fuertemente afectado ya que se tendrían dos periodos del año adecuados para el aprovechamiento de los residuos y habría escasez de sustratos para mantener la operación del sistema (Figura 5).

\section{Ajustes al potencial encontrado}

Un aspecto crucial para la obtención del potencial de metano es la temperatura del proceso, esta se puede ver afectada por la localización del sistema.
Respecto a esto, las temperaturas en estos municipios fluctúan entre 12 y $30^{\circ} \mathrm{C}$ lo cual está por fuera de los rangos óptimos de la digestión anaerobia. Sin embargo, mientras los valores de temperatura se puedan mantener entre 35 y $50^{\circ} \mathrm{C}$, los rendimientos de la producción de metano pueden mantenerse similares, lo que indica que es posible que el proceso requiera calefacción lo cual debe tenerse en cuenta en el balance energético y en el estudio de viabilidad de dichas plantas [49]. Sumado a esto, se debe tener en cuenta, para acotar este potencial, la competencia que se genera por los residuos para mantener las propiedades del suelo, forraje, otros tipos de aprovechamiento energético, la cobertura de suelos para evitar erosión, entradas orgánicas para soportar las comunidades en el suelo, propiedades y procesos de los ecosistemas [50]. Esto está ligado a usos que se dan actualmente a los residuos generados por las actividades pecuarias y agrícolas. En el tema de aporte de minerales o nutrientes a suelos, este requerimiento se podría cubrir utilizando los biosólidos obtenidos durante la digestión anaerobia como enmienda orgánica, lo cual tendría un doble valor agregado ya que sería un material estable con mejores propiedades que el residuo. Así mismo, la disponibilidad de los residuos es vital para la optimización de los sistemas buscando minimizar costos, transporte, almacenamiento y aspectos ambientales [16].

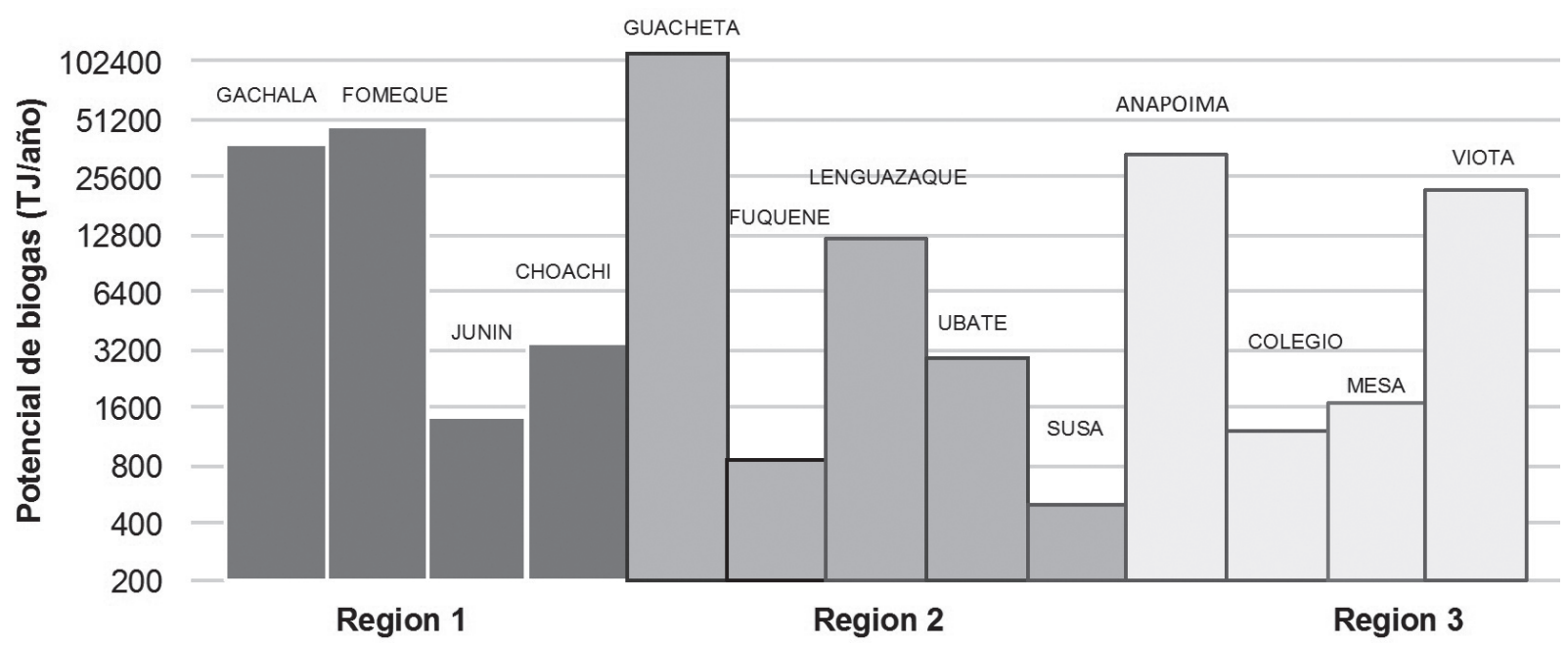

Figura 4. Aporte al potencial de metano de cada municipio por región. 


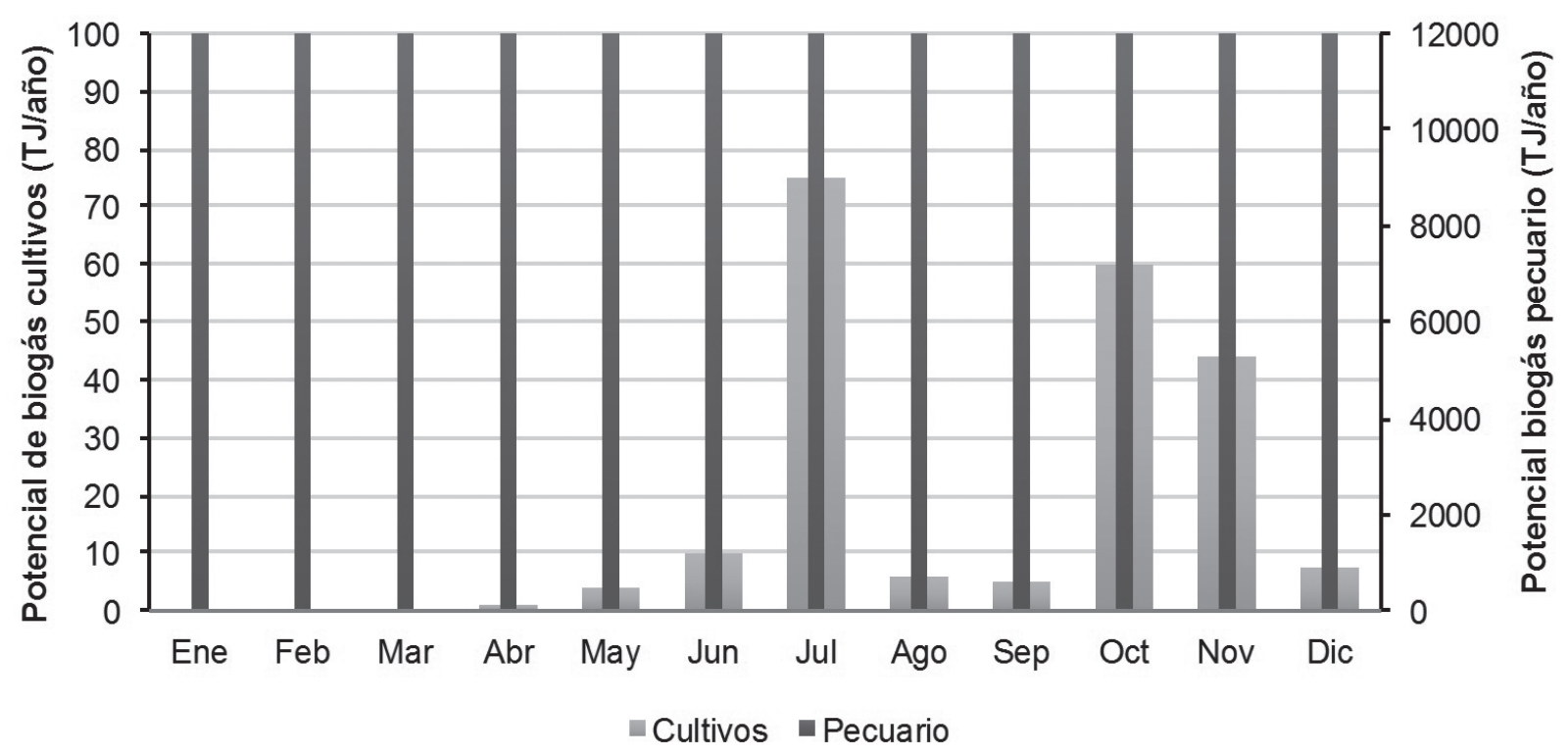

Figura 5. Evolución temporal del potencial de metano (biogás) estimado para la región 2.

El potencial establecido para municipios o regiones tendrá que tener en cuenta que la disponibilidad de diversos residuos requerirá un proceso de co-digestión anaerobia, donde el potencial de dicha mezcla podrá variar de acuerdo a las proporciones de los sustratos y las características físicoquímicas de cada uno [33]. Estudios han demostrado que la mezcla de residuos de alimentos con diferentes mezclas de estiércol resultaron en un aumento del rendimiento de producción de metano, en comparación con la digestión de estiércol o residuos de alimentos y de actividades pecuarias evaluados individualmente [51]. La producción anual de metano generado en diferentes plantas centralizadas utilizando sustratos como estiércol bovino, estiércol de aves de corral y pollos, residuos orgánicos e industriales es de $7,3 \times 10^{6} \mathrm{~m}^{3} / a n ̃ o$, donde gracias a esta mezcla se aumentó el BMP en un $80 \%$ [52]. Las mezclas realizadas generalmente utilizan estiércol principalmente de especies bovinas, residuos vegetales y residuos lignocelulósicos. En estos casos el potencial de dos plantas generadoras, de $2,4 \times 10^{5} \mathrm{~m}^{3} /$ año y $4,5 \times 10^{4} \mathrm{~m}^{3} / a n ̃ o$, se vio influenciado significativamente al agregar a la mezcla estiércol bovino lo cual produjo un aumento exponencial en la producción de biogás [42]. La adición de residuos de vegetales en un $2,5 \%$ de volumen y de residuos de granjas para estiércol de cerdo presenta un aumento de $124 \%$ en la producción de metano a diferencia del potencial obtenido con la digestión del estiércol por separado gracias a la reducción de la humedad al aumentar dicho residuo [40].

El aumento en la producción de biogás en las mezclas mencionadas se debe a que la presencia de estiércol produce un ajuste en la relación $\mathrm{C} / \mathrm{N}$, la cual es fundamental a la hora de producir biogás, ya que si esta relación es alta, el nitrógeno será consumido rápidamente por las bacterias metanogénicas presentes para formar proteínas y no reaccionará con el material restante, por lo tanto la producción de gas será alta. Lo contrario sucede si dicha relación es baja ya que el nitrógeno al ser la proporción mayor será liberado y acumulado en forma de amoniaco, el cual afectará el pH del biodigestor causando una acidificación del medio provocando una baja producción de gas, por ende dicha relación debe encontrarse entre 20/30 $[18,52,53]$. De acuerdo a esto, la co-digestión anaerobia de los residuos puede favorecer los resultados del potencial de biogás. Mientras que la temperatura del sistema se puede regular a través del uso del biogás que se produce en el proceso.

\section{Conclusiones}

El departamento de Cundinamarca tiene un potencial bruto de biogás de 1.117.567TJ/año producto de la transformación de los residuos agroindustriales. La distribución de los residuos generados en el departamento es heterogénea debido a la falta de tecnificación en varias de las actividades, principalmente agrícolas. Los residuos 
de mayor aporte en el potencial encontrado corresponden a aquellos alcanzados de actividades relacionadas con los cultivos de café, caña panelera, arveja, papa y excretas de bovinas y avícolas. Es importante tener en cuenta los ajustes que se deben plantear para los potenciales teóricos teniendo en cuenta actividades de competencia por los residuos, condiciones operativas del proceso, acceso a los residuos, características de los residuos de la región, generación de éstos y distancias entre los puntos de generación y planta centralizada. Por ende el mejor acercamiento para la realización de la evaluación del potencial del departamento de Cundinamarca es por medio de la identificación de dicho potencial por municipio debido a que se podrá tener en cuenta la generación de residuos, potencial y así mismo se tendrá en cuenta las distancias de recorrido desde el punto de generación hasta la planta de producción de biogás lo que permitirá realizar un análisis detallado de zonas aptas para la instalación de dicha planta, teniendo en cuenta los requerimientos básicos para su funcionamiento. El potencial de biogás analizado podrá variar de acuerdo a los sustratos que se mezclen en el proceso de co-digestión anaerobia, debido a que el potencial individual está influenciado por variables como relación $\mathrm{C} / \mathrm{N}$, relación de sólidos volátiles y sólidos totales, lignina y proteínas. Por último, las zonas que podrían cobijar sistemas centralizados, para el aprovechamiento de residuos, están en eje a los municipios de Guaduas, Guachetá y Anapoima con potenciales cercanos a los reportados para plantas de biogás en varias regiones del mundo, donde registran una potencia instalada entre $9.421,2$ - 130.780TJ/año.

\section{Referencias bibliográficas}

[1] Gobernación de Cundinamarca. Estadísticas agropecuarias. Volumen 23. Secretaría de agricultura y desarrollo rural, editor. Cundinamarca: Gobernación de Cundinamarca; 2013. p. 3-525.

[2] Gobernación de Cundinamarca. Caracterización general de Cundinamarca. Cundinamarca; 2010 p. 3-337.

[3] Gobernación de Cundinamarca. Lineamientos de política de residuos sólidos para Cundinamarca. Cundinamarca; 2012 p. 1-9.

[4] Mata-Alvarez J, Dosta J, Romero-Güiza MS, Fonoll X, Peces M, Astals S. A critical review on anaerobic co-digestion achievements between
2010 and 2013. Renewable and Sustainable Energy Reviews. 2014;36:412-27.

[5] AINIA centro tecnológico. Co-digestión anaerobia. Valencia,España; 2008 p. 1-2.

[6] Agencia andaluza de la energía. Estudio básico del biogás. Andalucia, España; 2011 p. 3-166.

[7] Superintendencia de servicios. Disposición Final de Residuos Sólidos en Colombia 2013. Quinta edi. Bogotá; 2013. p. 1-61.

[8] Henao GJ. Aprovechamiento de los residuos orgánicos en Colombia. Universidad de Antioquía; 2008. p. 3-116.

[9] Metropolitana Valle de Aburra. Manual de Compostaje. primera ed. Asociación Colombiana de Ingeniería Ambiental y Sanitaria, editor. Valle de Aburra: Asociación Colombiana de Ingeniería Ambiental y Sanitaria; 2010. p. 82-8.

[10] Cervi RG, Esperancini MST, Bueno ODC. Viabilidad Económica de la Utilización de Biogás para la Conversión en Energía Eléctrica. Información tecnológica. 2011;22(4):3-14.

[11] Castillo EF, Cristancho DE, Arellano VA. Anaerobic digestion of urban solid waste. A study of operational conditions for anaerobic digestion of solid urban waste. Revista colombiana de Biotecnología. 2003;2:11-22.

[12]DANE. Encuesta agropecuaria. Bogotá, Colombia; 2013 p. 1-10.

[13]Ministerio de transporte. Sistema de información de costos eficientes para el transporte automotor de carga. 2015 p. 1-3.

[14]Mariscal G. Tratamiento Excretas Cerdos. FAO, Prod. Porc., pp. 1-9, 2007.

[15] Martinez C. Volumen de biodigestores. Cuba Sol., p. 1-5, 2006.

[16] Kumarappan S. Spatially and Temporally Optimal Biomass Procurement Contracting for Biorefineries. 2014;9:2069-89.

[17] Gobernación de Cundinamarca. Estadísticas agropecuarias. Estadísticas de Cundinamarca. Tercera ed. Colombia; 2013. p. 3-90.

[18] Li Y, Zhang R, Liu G, Chen C, He Y, Liu X. Comparison of methane production potential, biodegradability, and kinetics of different organic substrates. Bioresource technology. 2013;149:565-9.

[19] Qiao W, Yan X, Ye J, Sun Y, Wang W, Zhang Z. Evaluation of biogas production from different biomass wastes with/without hydrothermal pretreatment. Renewable Energy. 2011;36(12):3313-18. 
[20] Parra B. Producción de metano a partir de la digestión anaerobia de biorresiduos de origen municipal. Universidad del Valle; 2014. p. 1-79.

[21] Organización de las Naciones Unidas para la Alimentación y la Agricultura. El manejo de los residuos de cultivos, de los cultivos de cobertura y de la rotación de cultivos. Valencia,España; 2008 p. 1-26.

[22] Krishania M, Kumar V, Vijay VK, Malik A. Analysis of different techniques used for improvement of biomethanation process: A review. Fuel. 2013;106:1-9.

[23] Rodriguez N, Zambrano D. Los subproductos del café: fuente de energía renovable. Avances Técnicos Cenicafé. 2010;3:1-8.

[24] Romero R, Mamani H. Obtención de biogás como fuente de energía renovable a partir de los subproductos del café. Revista Investigaciones Altoandinas. 2013;15(2):241-52.

[25] Casas E, Orrego E, Acevedo A, Giraldo S. Biomasa de residuos agricolas en el departamento de Antioquía. Segunda edi. Ramirez CA, editor. Medellín-Antioquía: Universidad Pontificia Bolivariana; 2010. p. 3-117.

[26] Dupri I. Estimación de los residuos agrícolas generados en la isla de Tenerife. TenerifeEspaña; 2006 p. 3-20.

[27] Martinez A. Diseño de un modelo semiempírico de co-digestión anaerobia. Universidad de Zaragoza; 2014. p. 1-41.

[28] Probiogas PSE. Manual de Estado del Arte de la Co-digestión Anaerobia de Residuos Ganaderos y Agroindustriales. BarcelonaEspaña; 2009 p. 1-55.

[29] Song Z, Zhang C, Yang G, Feng Y, Ren G, Han $X$. Comparison of biogas development from households and medium and large-scale biogas plants in rural China. Renewable and Sustainable Energy Reviews. 2014;33:204-13.

[30] Subsecretaria de desarrollo rural. Utilización de estiércoles. Ciudad de México, México; 2010 p. 1-8.

[31] Herrero J, Alvarez R, Cespedes R, Rojas MR, Conde V, Aliaga L, et al. Cow, sheep and Ilama manure at psychrophilic anaerobic co-digestion with low cost tubular digesters in cold climate and high altitude. Bioresource Technology. 2015;181:238-46.

[32] iang Y, Heaven S, Banks CJ. Strategies for stable anaerobic digestion of vegetable waste. Renewable Energy. 2012;44:206-14.

[33] Garcia M, Gomez X. Anaerobic digestion of livestock wastes:vegetales residues co.substrate and digestate pot treatment. Universidad de Valladolid; 2010. p. 11-170.

[34] Mac S, Llabr P. Anaerobic digestion of organic solid wastes. An overview of research achievements and perspectives. Bioresource Technology. 2000;74(1):3-16.

[35] Organización de las Naciones Unidas para la Alimentación y la Agricultura (FAO), Manual De Biogás, Santiago de Chile, 2011.

[36] Corredor Becerra OF. Evaluación del potencial energético de la biomasa residual proveniente de cultivos energéticos. Bucaramanga, Colombia: Universidad Industrial de Santander; 2008. p. 7.

[37] Agencia andaluza de la energía. Estudio básico del biogás. Sevilla, España: 2011.

[38] Cadavid L, Bolaños I. Aprovechamiento de residuos orgánicos para la producción de energía renovable en una ciudad colombiana. Energética. 2015;(46):23-8.

[39] Quintero M. Estudio preliminar para la producción de biogás a partir de la digestión anaerobia de mucílago de café. BUcaramanga, Colombia: Universidad Industrial de Santander; 2012. p. 1-59.

[40] Rodríguez Valencia N. Manejo de residuos en la agroindustria cafetera. Chinchina Caldas, Colombia; 2000. p. 1-10.

[41] Martinez Hernández CM, Oechsner $H$, Brulé $M$, Marañon Maison E. Estudio de algunas propiedades físico-mecánicas y químicas de residuos orgánicos a utilizar en la producción de biogás en Cuba. (Spanish). Stud. some Phys. Chem. Prop. Org. waste to use biogas Prod. Cuba. 2014;23(2):63-914.

[42] Pascual A, Ruiz B. Situación y potencial de generación de biogás. Primera ed. Instituto para la diversificación y ahorro de energía, editor. Madrid, España: Instituto para la diversificación y ahorro de energía; 2011. p. 1-104.

[43] Mang HP, Huba EM. Co-digestion: Some European Experiences Co-digestión : Algunas experiencias Europea. Alemania: Fördergesellschaft für nachhaltige Biogas- und Bioenergienutzung; 2011 p. 25-59.

[44] Igliński B, Buczkowski R, Cichosz M. Biogas production in Poland-Current state, potential and perspectives. Renewable and Sustainable Energy Reviews. 2015;50:686-95.

[45] Montenegro M. Biogás: una alternativa ecológica para la producción de energía. Biomass Bioenergy. 2012;7(85):881-94. 
[46] Infantes P. Diseño y Construcción de un Biodigestor Industrial para tratamiento de residuos orgánicos. Arequipa, Perú; 2010 p. 3-55.

[47]Fuchsz M, Kohlheb N. Comparison of the environmental effects of manure- and cropbased agricultural biogas plants using life cycle analysis. Journal of Cleaner Production. 2015;86:60-6.

[48] Fantin V, Giuliano A, Manfredi M, Ottaviano G, Stefanova M, Masoni P. Environmental assessment of electricity generation from an Italian anaerobic digestion plant. Biomass and Bioenergy. 2015;83:422-35.

[49] Lindorfer H, Waltenberger R, Köllner K, Braun $\mathrm{R}$, Kirchmayr R. New data on temperature optimum and temperature changes in energy crop digesters. Bioresource technology. 2008;99(15):7011-9.

[50] Franzluebbers AJ. Farming strategies to fuel bioenergy demands and facilitate essential soil services. Geoderma. 2015;259-260:251-8.

[51]Lisboa MS, Lansing S. Characterizing food waste substrates for co-digestion through biochemical methane potential (BMP) experiments. Waste management. 2013;33(12):2664-9.

[52] Larrahondo JE. Calidad de la Caña de Azúcar. In: Cenicaña, editor. Cultivo de caña. primera ed. Cali, Valle del Cauca; 2008. p. 337-55.

[53] Unidad minero energética. Guía para la implementación de sistemas para la producción de biogás. Bogotá, Colombia; 2003 p. 1-47. 